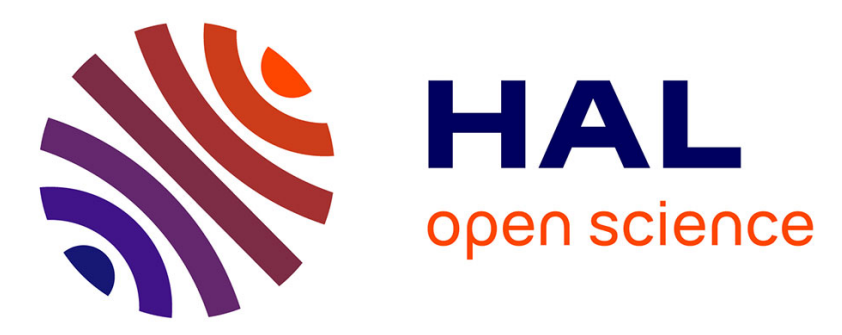

\title{
Role of robot-assisted laparoscopy in adjuvant surgery for locally advanced cervical cancer
}

Eric Lambaudie, Fabrice Narducci, Marie Bannier, Camille Jauffret, Nicolas Pouget, Eric Leblanc, Gilles Houvenaeghel

\section{- To cite this version:}

Eric Lambaudie, Fabrice Narducci, Marie Bannier, Camille Jauffret, Nicolas Pouget, et al.. Role of robot-assisted laparoscopy in adjuvant surgery for locally advanced cervical cancer. EJSO - European Journal of Surgical Oncology, 2010, 36 (4), pp.409. 10.1016/j.ejso.2009.12.004 • hal-00576159

\section{HAL Id: hal-00576159 \\ https://hal.science/hal-00576159}

Submitted on 13 Mar 2011

HAL is a multi-disciplinary open access archive for the deposit and dissemination of scientific research documents, whether they are published or not. The documents may come from teaching and research institutions in France or abroad, or from public or private research centers.
L'archive ouverte pluridisciplinaire HAL, est destinée au dépôt et à la diffusion de documents scientifiques de niveau recherche, publiés ou non, émanant des établissements d'enseignement et de recherche français ou étrangers, des laboratoires publics ou privés. 


\section{Accepted Manuscript}

Title: Role of robot-assisted laparoscopy in adjuvant surgery for locally advanced cervical cancer

Authors: Eric Lambaudie, Fabrice Narducci, Marie Bannier, Camille Jauffret, Nicolas Pouget, Eric Leblanc, Gilles Houvenaeghel

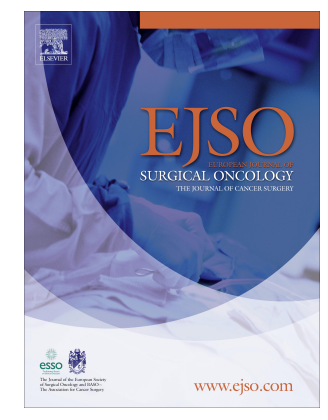

PII:

S0748-7983(09)00548-4

DOI:

10.1016/j.ejso.2009.12.004

Reference: YEJSO 2927

To appear in: European Journal of Surgical Oncology

Received Date: 26 April 2009

Revised Date: 15 December 2009

Accepted Date: 17 December 2009

Please cite this article as: Lambaudie E, Narducci F, Bannier M, Jauffret C, Pouget N, Leblanc E, Houvenaeghel G. Role of robot-assisted laparoscopy in adjuvant surgery for locally advanced cervical cancer, European Journal of Surgical Oncology (2009), doi: 10.1016/j.ejso.2009.12.004

This is a PDF file of an unedited manuscript that has been accepted for publication. As a service to our customers we are providing this early version of the manuscript. The manuscript will undergo copyediting, typesetting, and review of the resulting proof before it is published in its final form. Please note that during the production process errors may be discovered which could affect the content, and all legal disclaimers that apply to the journal pertain. 


\section{Role of robot-assisted laparoscopy in adjuvant surgery for locally advanced cervical cancer}

Eric Lambaudie ${ }^{\mathrm{a}}$, Fabrice Narducci ${ }^{\mathrm{b}}$, Marie Bannier ${ }^{\mathrm{a}}$, Camille Jauffret ${ }^{\mathrm{a}}$, Nicolas Pouget $^{\mathrm{a}}$ Eric

$$
\text { Leblanc }^{\mathrm{b}} \text {, Gilles Houvenaeghel }{ }^{\mathrm{a}}
$$

${ }^{a}$ Paoli Calmettes Institute, Department of Surgery, 232 bd Sainte Marguerite, 13009 Marseille, France

${ }^{\mathrm{b}}$ Oscar Lambret Center, Department of Surgery, 3 rue Frédéric Combemale, BP307 59020 Lille cedex France, 59000 Lille, France

\section{Corresponding author:}

Eric Lambaudie

Paoli Calmettes Institute

232 Bd Sainte Marguerite

13009 Marseille

France

Phone: + 334912235 32; Fax: + 334912236 13; Email: lambaudie@ marseille.fnclcc.fr 


\section{Abstract}

Objective. The aim of this study was to compare the feasibility and efficacy of robot-assisted laparoscopy with traditional laparotomy and conventional laparoscopy in a series of patients with locally advanced cervical cancer managed in our two institutions.

Methods. Twenty-two patients who underwent robot-assisted laparoscopy were compared with 20 patients who underwent adjuvant surgery by laparotomy and 16 who underwent conventional laparoscopy, before the arrival of the Da Vinci surgical system.

Results. There was no significant difference between the three groups in terms of body mass index, FIGO stage, or tumor histology. The complication rate was similar in the three groups of patients, although there was a trend towards more lymphatic complications in the robotassisted subgroup managed medically. There was no significant difference in the recurrence rate between the robot-assisted laparoscopy, conventional laparoscopy and laparotomy groups $(27.3 \%, 29.4 \%$ and $30 \%$, respectively).

Conclusion. Robot-assisted laparoscopy is feasible after concurrent chemoradiation and brachytherapy in cases of locally advanced cervical cancer. This new surgical approach reduces hospital stay, and seems to result in less severe complications than conventional laparotomy without modifying the oncological outcome.

Keywords: Locally advanced cervical cancer; Adjuvant surgery; Robot-assisted laparoscopy 


\section{Introduction}

Chemoradiation is recognized as the standard treatment for locally advanced cervical cancer [1]. Whenever technically possible, chemoradiation should be complemented by adjuvant brachytherapy. If residual cervical tumors or lymph node involvement are observed, adjuvant surgery (or "end" surgery) is favored to optimize local control [2, 3].

Since the late 1990s, robotic assistance (Da Vinci; Intuitive Surgical) has found an important place in mini-invasive surgical procedures. Recently, various applications have been described in gynecological malignancies: endometrial cancer staging, radical hysterectomy [4], trachelectomy [5], and lymph node staging via the trans-peritoneal or extraperitoneal route $[6,7]$.

We report our experience of this procedure in a subgroup of patients managed for advanced cervical cancer, after carrying out 100 robotic surgical gynecological procedures in our two cancer centers. To our knowledge, this is the first report to describe the use of this procedure in oncology patients, because "end surgery" is an option in advanced cervical cancer management and because robotic assistance is a new surgical technique. All of our patients were managed by experienced oncological surgical teams, especially with regard to conventional laparoscopy.

The aim of this study was to demonstrate the feasibility of robot-assisted laparoscopy in comparison to traditional laparotomy and conventional laparoscopy in a series of patients with locally advanced cervical cancer.

\section{Patients and Methods}

All the procedures has been performed by 4 surgeons, in our 2 institutions

\section{Robot-assisted laparoscopy patients}


Twenty-two patients underwent robot-assisted laparoscopy. Peri- and post-operative data were recorded prospectively, including operation time from skin incision to skin closure, and installation and docking time for the Da Vinci surgical system. Blood loss, peri- and postoperative morbidity, length of hospitalization (from the day of surgery), and histological results were also recorded.

Data concerning the characteristics of the patients, the surgical procedure, histological findings and follow-up were also collected.

\section{Surgical procedure}

Since the arrival of Da Vinci S in our oncological surgical departments (Marseille and Lille, France) in February 2007, adjuvant surgery in patients referred for advanced cervical cancer has been carried out laparoscopically with robot assistance.

All patients were placed in the low lithotomy position with their arms padded and tucked to the side. The Da Vinci unit was positioned between the legs for all pelvic procedures, with or without lombo-aortic exploration. In all cases, five ports were placed: four for the Da Vinci surgical system's arms (one camera port, three instrument ports) and the fifth as a classical laparoscopic port for the assistant (suction, specimen removal, needle application, etc).

The first port was placed after opening the abdominal cavity with a small abdominal incision to introduce the camera. The position of the camera depended on the anatomical site of the intended procedure. For pelvic surgery, the camera port was placed $1-2 \mathrm{~cm}$ above the umbilicus and the four additional ports were positioned in a curved line, keeping a $7-8 \mathrm{~cm}$ distance between the ports. After routine exploration of the peritoneal cavity, the Da Vinci unit was docked.

In the case of locally advanced cervical cancer (IB2, II, III), treatment included lomboaortic lymph node staging prior to concurrent chemoradiation to define the radiation field, 
brachytherapy and surgery (simple colpohysterectomy + lymphadenectomy). Radical hysterectomy was performed only if difficulties were encountered during the surgical procedure in exposing surgical landmarks (ureter, uterine pedicle) due to fibrosis and retraction after concomitant chemoradiation and brachytherapy.

\section{Traditional laparotomy and laparoscopy patients}

Two groups of patients underwent the same treatment with adjuvant surgery performed by laparotomy $(n=20)$ or conventional laparoscopy $(n=16)$. Before the arrival of the Da Vinci robotic system, the choice of surgical approach depended on the surgeon's previous laparoscopy experience. However, if a bulky residual tumor was suspected on MRI after chemoradiation, laparotomy was the procedure of choice. In the conventional laparoscopic subgroup, radical hysterectomy was performed systematically.

\section{Statistical analysis}

Statistical analysis was performed using the Kruskall Wallis test and Chi-square test. A pvalue of 0.05 was considered statistically significant. All statistical analyses were performed using SPSS 12 software for Windows. Differences in the survival curves or in the disease-free survival curves were estimated by log-rank test.

\section{Results}

\section{Demographic and clinical characteristics of the patients}

The characteristics of the 58 patients included in the study are shown in Table 1. There was no significant difference between the three groups in terms of body mass index, FIGO stage, or tumor histology. Two cases of FIGO stage IB1 and one case of FIGO stage IA2 were included as advanced cervical cancer because of pelvic lymph node involvement diagnosed surgically (laparoscopic lymph node staging before brachytherapy).

\section{Comparison of surgical procedures}


In terms of per-operative criteria, a significant difference was observed between robotassisted laparoscopy, conventional laparoscopy and laparotomy in terms of operative time; conventional laparoscopy took longer than robot-assisted laparoscopy and laparotomy in this indication (Table 2). This can be explained by the surgical approach used, which consisted of systematic radical hysterectomy in the subgroup treated by conventional laparoscopy.

On the other hand, a significant difference was observed in hospital stay between laparoscopy (robot-assisted and conventional) and laparotomy patients, while there was no difference between robot-assisted laparoscopy and conventional laparoscopy. No conversion was necessary in the two laparoscopic groups and blood transfusions were necessary in only two cases (one in the robotic group, one in the laparotomy group).

There was no significant different in complication rate between the three groups of patients (Table 2). However, there was a trend towards more lymphatic complications in the robot-assisted subgroup managed medically (painkillers) or with drains (two cases).

\section{Histological findings and follow-up}

The histological findings in the 56 patients are summarized in Table 3; there was no significant difference between the three groups, although residual cervical tumor was more frequent in the laparotomy group (patient selection after chemoradiation, with suspected bulky residual tumor). There was also no difference in median number of lymph nodes removed between the three groups.

Follow-up was significantly higher with conventional laparoscopy and laparotomy. Since the arrival of Da Vinci, all surgical indications previously managed by the traditional route are now managed robotically. There was no difference in recurrence rate between robotassisted laparoscopy, conventional laparoscopy and laparotomy $(27.3 \%, 29.4 \%$ and 30\%, respectively. The time interval between surgery and recurrence was also similar in the three 
groups. Because of the short follow up, we did not observe significative difference in term of overall survival and disease-free survival.

\section{Discussion}

The benefit of adjuvant surgery in the management of locally advanced cervical cancer is debatable. Only one randomized GOG study reported lower relapse- and higher progression-free survival in patients treated with radiation and surgery. Overall survival was similar in the two groups and all patients were treated with concurrent chemoradiation. Surgery-associated morbidity is high: about 10\% Grade 3-4 morbidity described in the randomized trial of Keys et al. [8].

In a previous study, residual cervical tumors were observed in $40-50 \%$ of patients and pelvic positive lymph nodes in $16 \%$ [9]. In the current series, these data were not recorded since only two stage IIIs and one stage IV were observed in the three groups. It is known that that the rate of residual disease increases with FIGO stage: $43-52 \%$ in stage IB2, 41-56\% in stages IIA and IIB, 51.6\%-68\% in stage III, and 72.7-73.7\% in stage IVA [2,10,11].

In our experience, and as reported previously, stages III and IV seem to have an impact in terms of mortality but with no significant difference concerning overall survival compared to stages I and II [12]. In patients who do not respond to chemoradiation, in the absence of para-aortic involvement, $80 \%$ of patients survive to 5 years after curative surgery [9].

This argument justifies our therapeutic strategy and the absolute necessity to reduce the complication rate or grade. Mini-invasive surgery seems to be an attractive option and constitutes a new surgical field for robotically-assisted laparoscopy.

Several publications have reported on the feasibility of robot-assisted laparoscopy in cervical cancer: lymph node staging, radical hysterectomy, trachelectomy $[4,5,13]$, and the 
first case of anterior pelvic exenteration has also been reported (Lambaudie et al., Gynecol Oncol 2009 in Press). Thus, there is no doubt about the feasibility of extrafascial or radical hysterectomy after chemoradiation, as described in this study. Indeed, 3-dimensional vision, tremor filtration and the precision and maneuverability of robotic instruments are advantages that assist in easier dissection, particularly in the case of fibrosis or tissue retraction after chemoradiation.

Boggess et al. [4], Magrina et al. [7] and Sert et al. (14) in their respective series of radical hysterectomy observed significant difference between robot-assisted laparoscopy, conventional laparoscopy and laparotomy in terms of operation time, mean blood loss, hospital stay and histological findings (particularly quality of lymph node dissection).

As in the current series, Nezhat [15] did not observe any significant differences in peroperative criteria or histological findings between two groups of patients who underwent laparoscopic robot-assisted radical hysterectomy $(n=13)$ or conventional laparoscopic radical hysterectomy $(n=30)$. The conclusion of this prospective experience was that despite the multiple advantages of robotic assistance published in the literature, robot-assisted procedures have no advantages compared to traditional total laparoscopic radical hysterectomy when performed by an experienced laparoscopic surgeon.

Robot-assisted laparoscopy a major evolution of laparoscopy. Robot-assisted laparoscopy and conventional laparoscopy are similar in terms of operation time (without docking time), hemorrhagic complications, median number of resected lymph nodes and hospital stay. Difference concerning lymphatic complications has to be confirmed. We think that it is due to the greater interest of this type of complications since almost 5 years (but not to surgical technique which is not different in our 3 groups concerning lymph node dissection), which has an impact on quality of life. 
However, in this study, surgical teams were experienced in conventional laparoscopy. In an experienced oncological surgeon's hands, robot-assisted laparoscopy, with a shorter learning curve, should develop for the patients' benefit (little scarring, lower hemorrhagic complications, and shorter hospital stay). The real advantage of robot-assistance is its application to advanced laparoscopic surgical procedures with less training than for conventional laparoscopy.

Reynolds and Advincula [16] suggested a cutoff at 30 patients to obtain a similar complication rate and operation time for robot-assisted hysterectomy compared to abdominal hysterectomy. We think that previous laparoscopic experience and the possibility of performing the first robotic procedure by a team of two surgeons should contribute to a reduced learning curve.

In conclusion, robot-assisted laparoscopy is feasible after concurrent chemoradiation and brachytherapy in cases of locally advanced cervical cancer. This new surgical approach reduces hospital stay, and is associated with a lower rate of serious complications than laparotomy without modifying the oncological outcome. In the hands of experienced oncological surgeons, robot-assisted laparoscopy with a shorter learning curve should develop for the patients' benefit (laparoscopic advantages: little scarring, lower hemorrhagic complications and shorter hospital stay).

\section{Conflict of Interest Statement}

Eric Lambaudie and Gilles Houvenaeghel are proctor for Intuitive Surgical The others authors declare that there are no conflicts of interest 


\section{References}

[1] J. Green, J. Kirwan, J. Tierney, C. Vale, P. Symonds and L. Fresco L et al., Concomitant chemotherapy and radiation therapy for cancer of the uterine cervix, Cochrane Database Syst. Rev. 3 (2005), CD002225.

[2] H.M. Keys, B.N. Bundy, F.B. Stehman et al., Cisplatin, radiation, and adjuvant hysterectomy compared with radiation and adjuvant hysterectomy for bulky stage IB cervical carcinoma, N. Engl. J. Med. 340 (1999), pp. 1154-1161.

[3] J.M. Classe, P. Rauch, J.F. Rodier, P. Morice, E. Stoeckle and S. Lasry et al., Surgery after concurrent chemoradiotherapy and brachytherapy for the treatment of advanced cervical cancer: morbidity and outcome: results of a multicentric study of the GCCLCC, Gynecol. Oncol. 102 (2006), pp. 523-529.

[4] J.F. Boggess, P.A. Gehrig, L. Cantrell et al., A case-control study of robot-assisted type III radical hysterectomy with pelvic lymph node dissection compare with open radical hysterectomy, Am. J. Obstet. Gynecol. 199 (2008), p. 357.

[5] J. Persson, P. Kannisto and T. Bossmar, Robot-assisted abdominal laparoscopic radical trachelectomy, Gynecol. Oncol. 111 (2008), p. 564.

[6] I. Vergote, B. Pouseele, T. Van Gorp et al., Robotic retroperitoneal lower para-aortic lymphadenectomy in cervical carcinoma: first report on the technique used in 5 patients, Acta. Obstet. Gynecol. Scand. 87 (2008), p. 783.

[7] J.F. Magrina, R.M. Kho, A.L. Weaver, R.P. Montero and P.M. Magtibay, Robotic radical hysterectomy: comparison with laparoscopy and laparotomy, Gynecol. Oncol. 109 (2008), pp. 86-91. 
[8] H.M. Keys, B.N. Bundy, F.B. Stehman et al., Radiation therapy with and without extrafascial hysterectomy for bulky stage IB cervical carcinoma: a randomized trial of the Gynecologic Oncology Group, Gynecol. Oncol. 89 (2003), pp. 343-353.

[9] G. Houvenaeghel, L. Lelièvre, M. Buttarelli, J. Jacquemier, X. Carcopino and P. Viens et $a l .$, Contribution of surgery in patients with bulky residual disease after chemoradiation for advanced cervical carcinoma. EJSO. 33 (2007), pp. 498-503.

[10] G. Houvenaeghel, V. Moutardier, F. Bladou and R. Zalta, Recurrences of pelvic cancers. Pelvic reconstruction: when, how, why? Ann. Chir. 53 (1999), pp. 85-98.

[11] J.M. Classe, P. Morice, J. Rodier et al., Pathological evaluation of pelvic radiotherapy with concurrent cisplatin-based chemotherapy followed by surgery in treatment of locally advanced cervical cancer: a retrospective multicentric experience of French Federation of Cancer Centers, Eur. J. Surg. Oncol. 28 (2002), p. 61.

[12] X. Carcopino, G. Houvenaeghel, M. Butarelli et al., Equivalent survival in patients with advanced stage IB-II and III-IVA cervical cancer treated by adjuvant surgery following chemoradiotherapy, EJSO. 34 (2008), pp. 569-575.

[13] E. Lambaudie, G. Houvenaeghel et al., Robot-assisted laparoscopy in gynaecologic oncology, Surg. Endosc. 22 (2008), p. 2743.

(14) Sert B. and Abeler V., Robotic radical hysterectomy in early-stage cervical carcinoma patients, comparing results with total laparoscopic radical hysterectomy cases. The future is now? Int J Med Robot, 2007. 3(3): p. 224-8.

[15] F. Nezhat, Minimally invasive surgery in gynaecologic oncology: laparoscopy versus robotics, Gynecol. Oncol. 111(suppl 2) (2008), S29.

[16] R.K. Reynolds and A.P. Advincula, Robot assisted laparoscopic hysterectomy: technique and initial experience, Am. J. Surg. 191 (2006), pp. 555-560. 
Table 1: Demographic characteristics and initial cervical cancer FIGO stage of patients undergoing robot-assisted laparoscopy, conventional laparoscopy and laparotomy

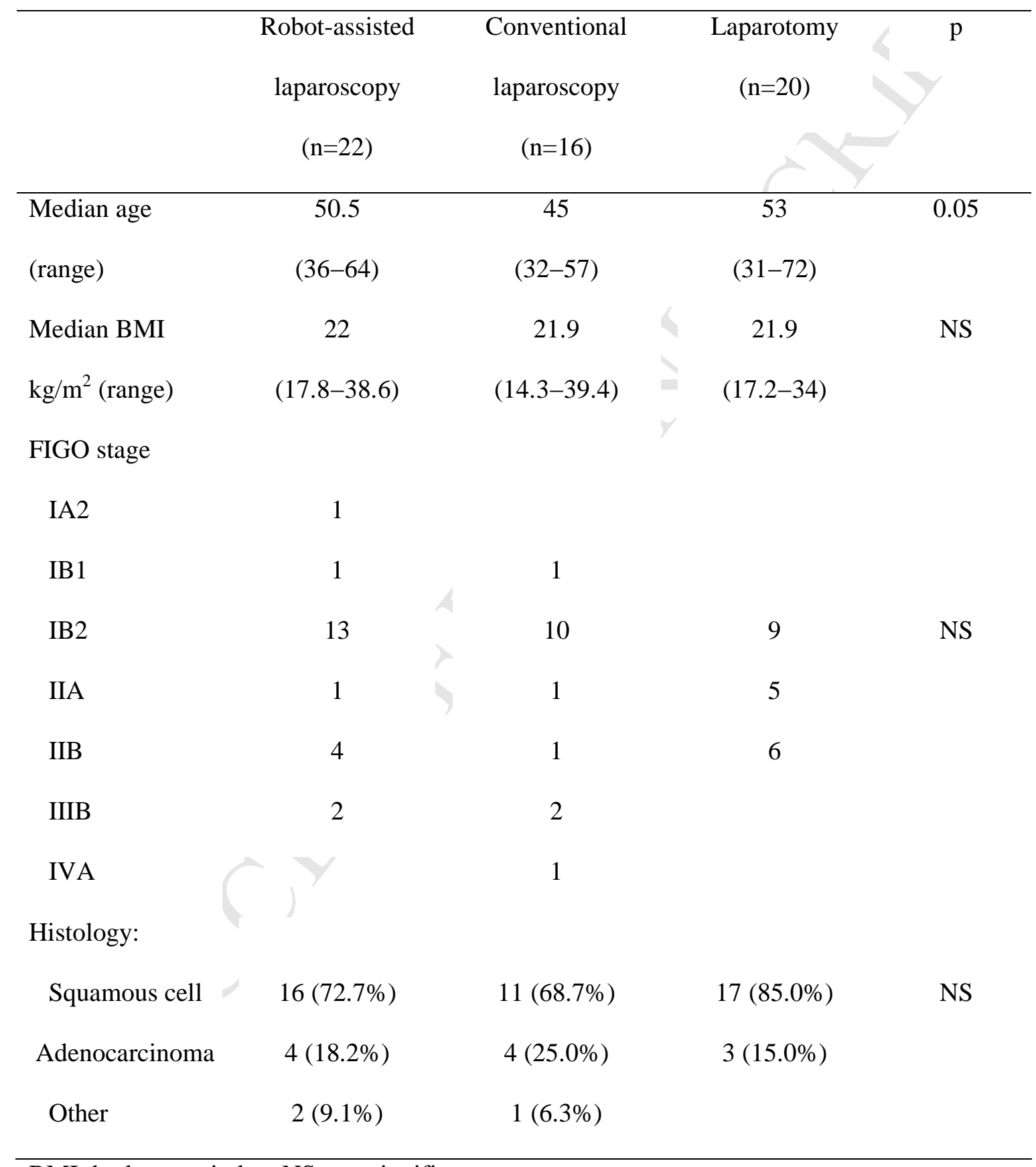

BMI: body mass index; NS: not significant 
Table 2: Comparison of operation time, transfusion rate, hospital stay and morbidity in patients undergoing robot-assisted laparoscopy, conventional laparoscopy and laparotomy

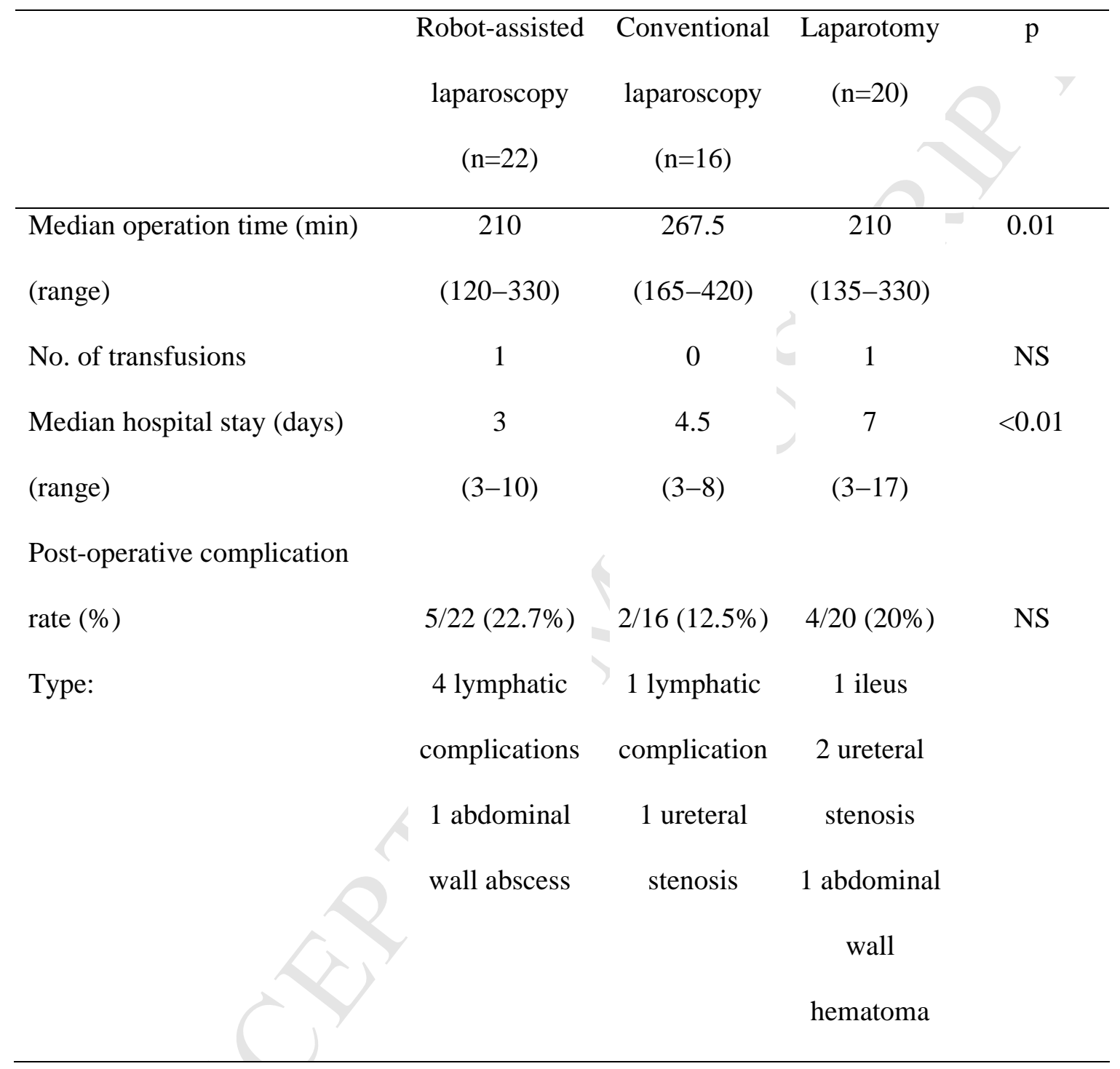


Table 3: Histological findings in patients undergoing robot-assisted laparoscopy, conventional laparoscopy and laparotomy

\begin{tabular}{|c|c|c|c|c|}
\hline & $\begin{array}{l}\text { Robot-assisted } \\
\text { laparoscopy } \\
(n=22)\end{array}$ & $\begin{array}{l}\text { Conventional } \\
\text { laparoscopy } \\
(n=16)\end{array}$ & $\begin{array}{l}\text { Laparotomy } \\
\qquad(\mathrm{n}=20)\end{array}$ & $\mathrm{p}$ \\
\hline \multicolumn{5}{|l|}{$>1 \mathrm{~mm}$ residual cervical tumor } \\
\hline$(\%)$ & $22.7 \%$ & $6.2 \%$ & $30 \%$ & NS \\
\hline+ & 5 & 1 & 6 & \\
\hline- & 17 & 14 & 14 & \\
\hline \multicolumn{5}{|l|}{ Median number of lymph nodes } \\
\hline $\begin{array}{l}\text { removed (right side/left side) } \\
\text { Residual lymph node }\end{array}$ & $5 / 4$ & $5 / 2.5$ & $6 / 6$ & NS \\
\hline involvement (\%) & $9.1 \%$ & $14.3 \%$ & $10 \%$ & NS \\
\hline+ & 2 & 2 & 2 & \\
\hline- & 20 & 14 & 18 & \\
\hline
\end{tabular}


Table 4: Follow-up, recurrences and mortality of patients undergoing robot-assisted laparoscopy, conventional laparoscopy and laparotomy

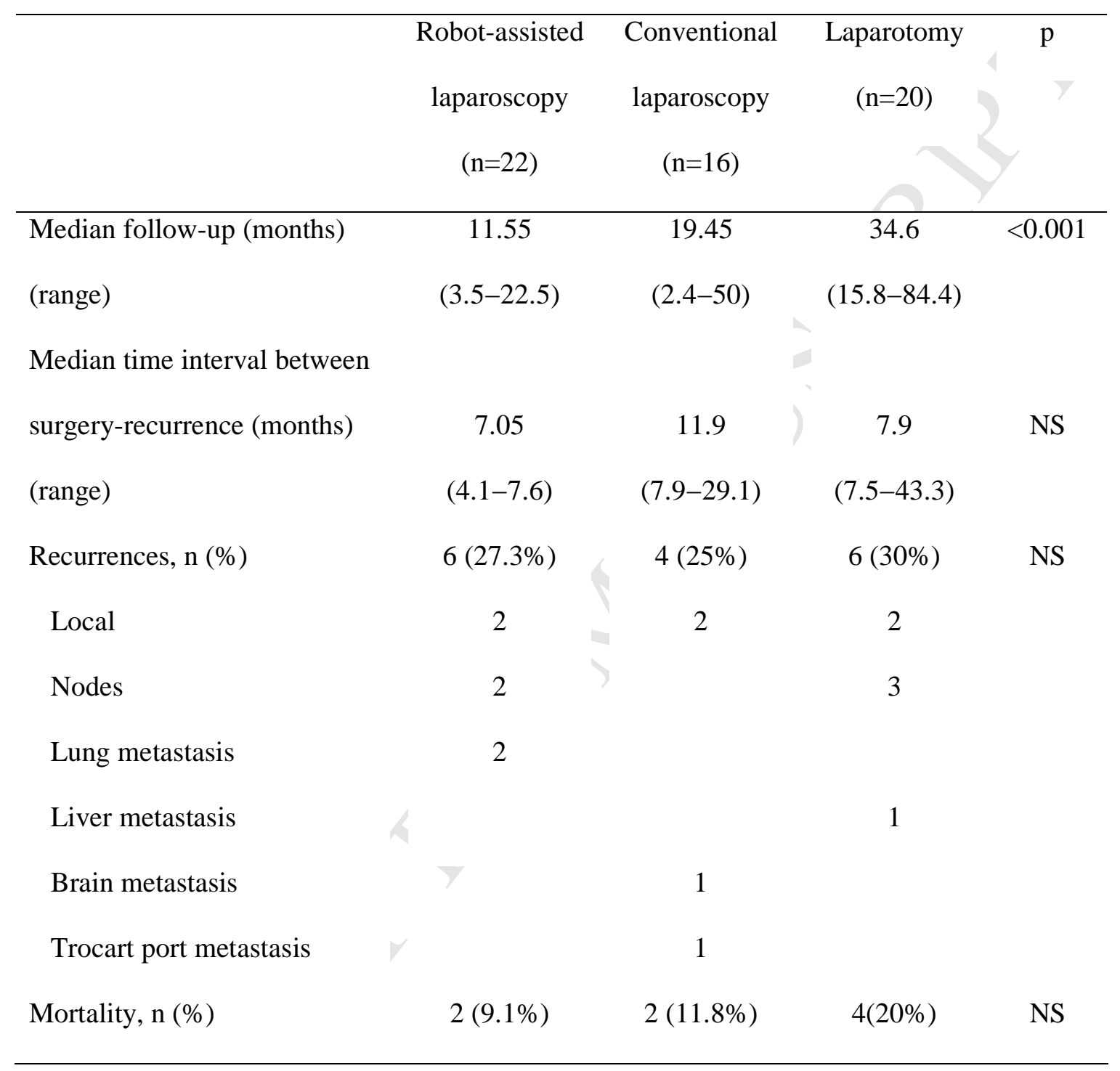

